\title{
ssssIn Situ, Operando Lithium K-edge Energy-loss Spectroscopy of Battery Materials
}

Wei Zhang ${ }^{1}$, Shanshan $\mathrm{YAO}^{2}$ and Feng Wang ${ }^{3}$

${ }^{1}$ Nankai University, Tianjin, Tianjin, China (People's Republic), ${ }^{2}$ Brookhaven National Lab, Rocky Point, New York, United States, ${ }^{3}$ Brookhaven National Laboratory, Upton, New York, United States

Development of next-generation safe, high energy lithium (Li)-batteries requires better understanding how electrodes function by characterizing electrochemical reaction and the associated ionic transport in anodes, cathodes and the involved interfaces/interphases. Such characterization is preferentially applied in the in situ, operando conditions, at relevant length scales. Most of the available techniques, such as those based on hard X-ray scattering, are suited for bulk measurement at electrode level, but very often have no adequate spatial resolution to probe local structural changes in single particles or interfaces [1]. Highresolution scanning/transmission electron microscopy (S/TEM) - electron energy-loss spectroscopy (EELS) is powerful, not only because of its high spatial resolution, but also the unparalleled analytical capability in imaging, diffraction, spectroscopy within a single instrument [2]. However, directly probing $\mathrm{Li}$, the "main player" in Li-batteries, poses a great challenge to traditional X-ray, electron diffraction and high-resolution S/TEM imaging due to its weak elastic scattering power and vulnerability to radiation damage. Here, we show that Li K-edge EELS can be a unique tool for probing Li, both of its spatial distribution and chemical state in the radiation-sensitive Li-battery materials [3-4], and may also be applied in situ, for tracking lithium ion transport in an operating battery electrode [5-6].

Recently, cryogenic S/TEM-EELS techniques were developed for characterization of the radiationsensitive Li-battery materials [7-8]. Alternatively, Li K-edge EELS spectroscopy may be applied for characterizing Li-containing materials, with potential benefits of reduced damage, because of the high ionization cross-section of the shallow Li K-edge. For example, the cross-section of Li K excitation (1s to $2 \mathrm{p}$ ) is 2 orders higher than that of the carbon (C) K (Figure 1a). With the optimized Li K-edge EELS, rich information about the electronic environment of the $\mathrm{Li}$ atoms may be obtained from the near-edge fine structure, allowing to differentiate between various lithium species (as illustrate in Figure 1b) [3]. Recently, an electrochemical cell specialized for operando Li K-edge EELS was developed [6]. As illustrated in Figure 2a, the cell was adapted from a TEM-grid based cell added with non-flammable ionic-liquid electrolyte, and has been applied for tracking $\mathrm{Li}^{+}$ion migration in lithium titanate $\left(\mathrm{Li}_{4} \mathrm{Ti}_{5} \mathrm{O}_{12}\right.$; LTO) nanoparticles. From the evolution of the EELS spectra (particularly in the pre-edge region), we were able to identify the local site occupancy and migration of $\mathrm{Li}^{+}$ions among different sites, thereby unveiling the kinetic pathways of lithium ion transport in fast charging LTO [6]. The opportunities for combining in situ, operando Li K-edge EELS with cryogenic S/TEM and other techniques, for complementary characterization of battery materials will also be discussed [9]. 

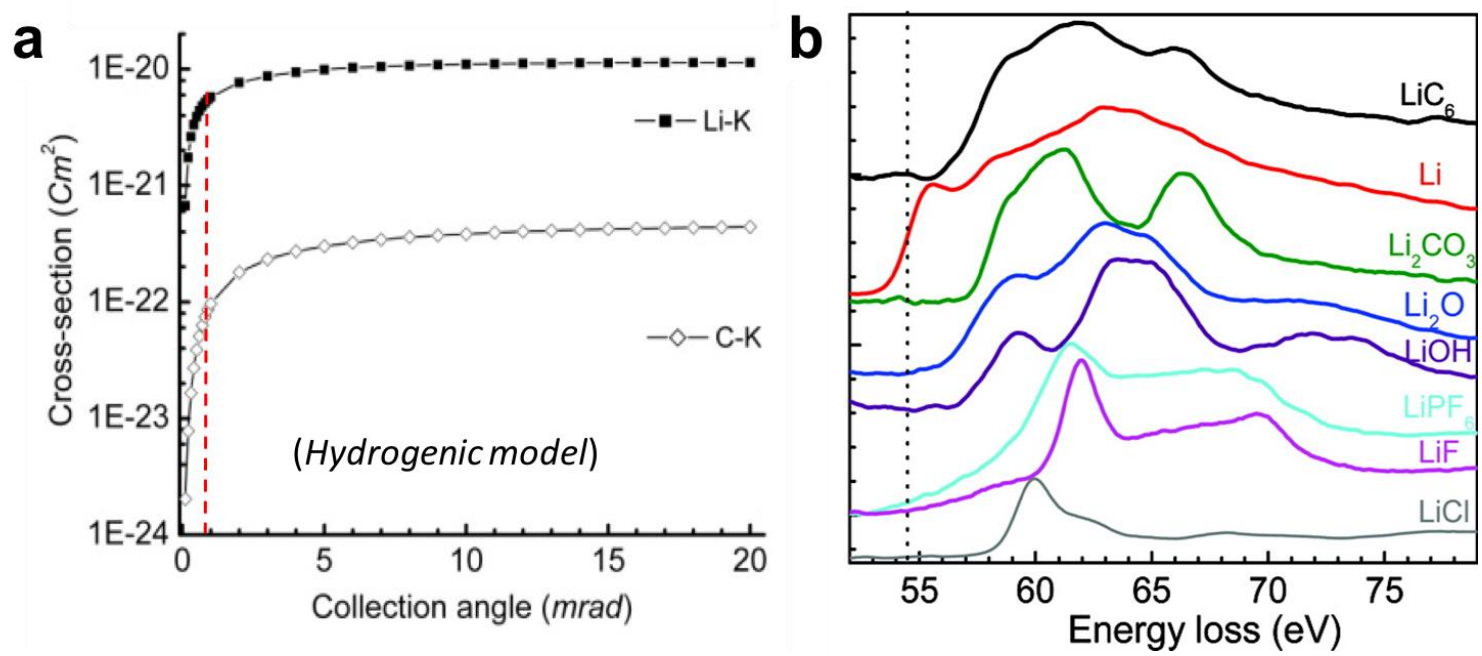

Figure 1. (a) Calculated inelastic scattering cross-section of the $1 \mathrm{~s}$ to $2 p$ transitions corresponding to the lithium and carbon K-edges, using a hydrogenic model. (b) Experimentally measured Li K-edge nearedge fine structure for a series of lithium compounds, viz., $\mathrm{Li}, \mathrm{LiC}_{6}, \mathrm{Li}_{2} \mathrm{CO}_{3}, \mathrm{Li}_{2} \mathrm{O}, \mathrm{LiOH}, \mathrm{LiPF} 6, \mathrm{LiF}$, and $\mathrm{LiCl}[3]$.
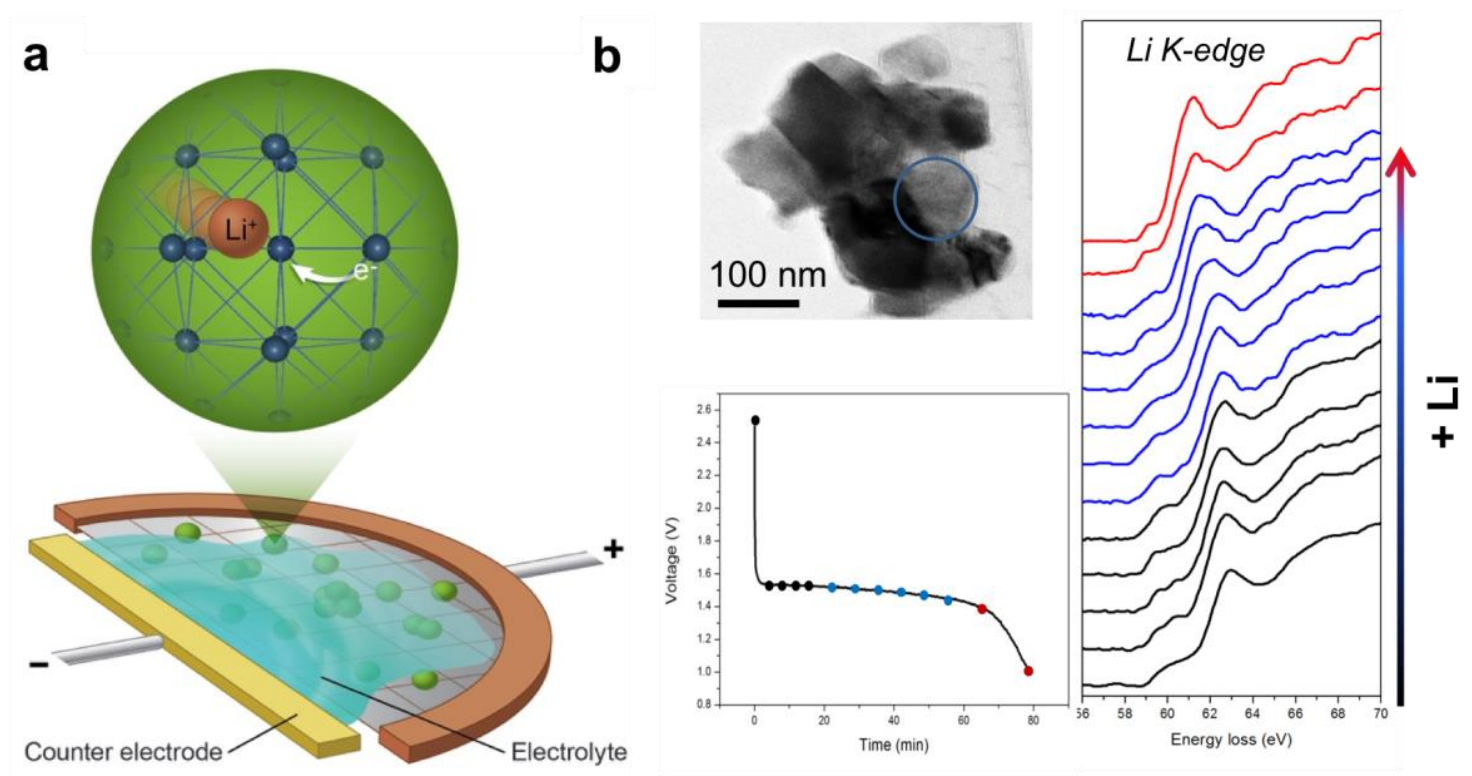

Figure 2. a) Schematic illustration of the configuration of electrochemical cell with special design for operando S/TEM-EELS measurements. (b) Time-resolved Li K-edge EELS spectra from Li4 $\mathrm{Ti}_{5} \mathrm{O}_{12}$ (LTO) nanoparticles during $1^{\text {st }}$ discharge, showing subtle but clear spectral changes in the pre-edge region, due to formation of metastable intermediates $\left(\mathrm{Li}_{4}+\mathrm{x} \mathrm{Ti}_{5} \mathrm{O}_{12}\right)$.

\section{References}

[1] H Wang, F Wang, "In-Situ, Operando Measurements of Rechargeable Batteries”, Current Opinion in Chemical Engineering 13, 170 (2016)

[2] R. F. Egerton, Electron energy-loss spectroscopy in the electron microscope. Springer Science \& Business Media (2011). 
[3] F. Wang et al., Chemical distribution and bonding of lithium in intercalated graphite: Identification with optimized electron energy loss spectroscopy. ACS nano 5, 1190 (2011).

[4] F. Wang et al., Excess lithium storage and charge compensation in nanoscale Li4+ xTi5O12. Nanotechnology 24, 424006 (2013).

[5] F. Wang, et al., "Tracking of Li Transport and electrochemical reaction in nanoparticles", Nat. Commun. 3, 1201 (2012).

[6] W. Zhang, et al., Kinetic Pathways of Ionic Transport in Fast Charging Lithium Titanate, Science. 367 (2020) 1030.

[7] Y. Li, et al., Atomic structure of sensitive battery materials and interfaces revealed by cryo-electron microscopy, Science 358, 506 (2017).

[8] M.J. Zachman, et al., Cryo-STEM mapping of solid-liquid interfaces and dendrites in lithium-metal batteries. Nature 560, 345 (2018).

[9] This work is financially supported partially by the Laboratory Directed Research and Development (LDRD) program at Brookhaven National Laboratory, and by part of the Center for Mesoscale Transport Properties, an Energy Frontier Research Center supported by the U.S. Department of Energy (DOE), Office of Science, Basic Energy Sciences, under Award DE-SC0012673, and by U.S. DOE Office of Energy Efficiency and Renewable Energy, Vehicle Technologies Office, Contract No. DE-SC0012704. 